\section{Prevalência de sobrepeso e obesidade em adolescentes de uma escola da rede pública do Recife}

\section{Overweight and obesity prevalence in adolescents from a public school in Recife}

Gisélia Alves Pontes da Silva 1

Geni Balaban 2

Eulália Maria M. Nascimento 3 Joana Darc Santana Baracho 4 Maria Maia V. Freitas 5

1,2 Departamento Materno Infantil, Centro de Ciências da Saúde da Universidade Federal de Pernambuco. Av. Moraes Rego, s/n. Cidade Universitária. Recife, Pernambuco, Brasil. CEP 50.670-420 3-5 Programa de Iniciação Científica. Universidade Federal de Pernambuco.

\begin{abstract}
Objectives: to determine the prevalence of overweight and obesity in students from a public school in Recife, Pernambuco, Brazil.

Methods: a cross sectional study was performed with 211 adolescents from a public school in Recife, in 2000. Overweight was defined as a Body Mass Index equal or above the 85th percentile and below the 95th percentile. Obesity was defined as a Body Mass Index equal or above the 95 th percentile.

Results: an overweight prevalence of $6,2 \%$ and an obesity prevalence of 5,7\% were found. There was not a statistically significant difference in overweight and obesity prevalences between males and females. Overweight prevalence was $6,6 \%$ in the male sex and $5,6 \%$, in females $(p=0,99)$. Obesity prevalence was $6,7 \%$ in female adolescents and $4,9 \%$ in males.

Conclusions: the overweight and obesity prevalences found in this study did not reach the magnitude observed in developed countries, but they deserve attention because of the risk of persistent obesity in adulthood and its association with several morbid conditions.
\end{abstract}

Key words Obesity, Adolescence, Prevalence

\section{Resumo}

Objetivos: determinar a prevalência de sobrepeso e obesidade em adolescentes de uma escola pública de Recife, Pernambuco, Brasil.

Métodos: realizou-se um estudo de corte transversal com 211 adolescentes de uma escola pública de Recife, no ano de 2000. Sobrepeso foi definido como Índice de Massa Corporal igual ou superior ao percentil 85 e inferior ao percentil 95, para idade e sexo. Obesidade foi definida como Índice de Massa Corporal igual ou superior ao percentil 95.

Resultados: as prevalências de sobrepeso e obesidade foram de 6,2\% e 5,7\%, respectivamente. Não houve diferença estatisticamente significante nas prevalências de sobrepeso e obesidade entre os sexos. A prevalência de sobrepeso no sexo masculino foi de $6,6 \%$ e, no feminino, $5,6 \%(p=0,99)$. A prevalência de obesidade foi de $6,7 \%$ no sexo feminino e de $4,9 \%$ no sexo masculino $(p=0,79)$.

Conclusões: as prevalências de sobrepeso e obesidade entre os adolescentes estudados não alcançaram a magnitude observada nos países desenvolvidos. Contudo, devem ser valorizadas, devido ao risco de obesidade persistente na idade adulta e às diversas condições mórbidas associadas à obesidade. Palavras-chave Obesidade, Adolescência, Prevalência. 


\section{Introdução}

A adolescência é um estágio do crescimento e desenvolvimento acompanhado de alterações morfofisiológicas complexas, nas quais a nutrição tem um papel fundamental. É nessa fase que o indivíduo adquire $25 \%$ de sua estatura final e $50 \%$ do seu peso definitivo, constituindo um período crítico para o potencial desenvolvimento de uma obesidade que venha a persistir na idade adulta. 1

A obesidade é um distúrbio nutricional e metabólico, o qual pode ser definido como um excesso de adiposidade no organismo. ${ }^{2}$ É uma doença de etiologia multifatorial, estando envolvidas causas genéticas e ambientais. 3

As conseqüências da obesidade na infância e adolescência incluem desde puberdade precoce, distúrbios psicossociais, como baixo rendimento escolar, isolamento e rejeição pelos colegas (estigmatização), até dislipidemias, hipertensão e aumento da mortalidade por doenças cardiovasculares na idade adulta. Diversos estudos têm demonstrado a associação de obesidade com o aumento da morbimortalidade experimentado pelos adultos que eram obesos quando adolescentes, fato esse que, em parte, independe do grau de obesidade do adulto. ${ }^{2-5}$

Nos Estados Unidos, a obesidade afeta entre 20 a $27 \%$ de todas as crianças e adolescentes, e $33 \%$ dos adultos. Nesse país, de 1976 a 1987, a prevalência da obesidade e da superobesidade (obesidade extrema, definida por alguns autores como IMC superior ao percentil $95 \mathrm{e}$, por outros, como peso relativo superior a $140 \%$ ), em jovens de 12 a 21 anos, aumentou em $64 \% .3$

Veiga et al. 6 encontraram uma prevalência de obesidade de $23 \%$ em adolescentes femininas de baixo nível sócioeconômico, no município de São Paulo, em 1992. Siqueri7 relata uma prevalência de sobrepeso de $13 \%$ em adolescentes do sexo feminino e de $18 \%$ nos adolescentes do sexo masculino, em inquérito realizado na cidade do Rio de Janeiro, em 1995 e 1996.

Apesar da definição de obesidade ser relativamente simples, é importante sua diferenciação com o termo sobrepeso. Esse último pode ser usado significando simplesmente um excesso ponderal para a estatura, enquanto que obesidade seria um termo reservado para situações em que a adiposidade é mensurada. Embora haja forte correlação entre peso elevado para estatura e obesidade medida em termos de adiposidade, a massa magra do corpo (músculos, ossos) também pode contribuir para o excesso ponderal. Em outras situações, o termo obesidade é reservado aos casos mais graves, enquanto sobrepeso refere-se aos mais leves. 8

Os critérios antropométricos para avaliação nutricional na adolescência são mais complexos, pois além de idade, peso e estatura, são também importantes os dados sobre o estágio de maturação sexual. Adolescentes de mesmo peso, estatura, idade e sexo podem encontrar-se em estágios diferentes do seu crescimento, o que dificulta a formação de padrões de referência. 6

Para o diagnóstico de sobrepeso e obesidade podemos utilizar o Índice de Massa Corporal (IMC), também denominado Índice de Quetelet. O IMC é uma medição indireta da gordura corpórea, obtida pela fórmula peso/estatura, que tem como vantagens a sua simplicidade, o fato de utilizar apenas dados que já são coletados rotineiramente na prática pediátrica, e a existência de padrões de referência internacionalmente aceitos para uma ampla faixa etária (6 a 74 anos). $9-12$

O Expert Committee on Clinical Guidelines for Overweight in Adolescent Preventive Services recomenda o uso do IMC para ajudar a definir obesidade no adolescente. ${ }^{13}$ Segundo o Comitê, um IMC igual ou superior ao percentil 95 para idade e sexo deve ser considerado sobrepeso, enquanto que um IMC igual ou superior ao percentil 85, mas inferior ao percentil 95 , seria indicativo de risco para sobrepeso. Must et al. 12 consideram os percentis $85 \mathrm{e}$ 95 do IMC como ponto de corte para definir obesidade e superobesidade, respectivamente. O IMC foi recomendado pela Word Health Organization $(\mathrm{WHO})^{8}$ como indicador de sobrepeso mais adequado na adolescência, usando como ponto de corte o percentil 85 para sexo e idade. A controvérsia nas definições de sobrepeso e obesidade dificulta a comparação das prevalências relatadas pelos diferentes autores. Portanto, ao comparar as prevalências encontradas nas diversas casuísticas, é necessário estar atento não só à nomenclatura, mas também aos pontos de corte utilizados por cada autor.

Considerando a crescente importância do tema obesidade, bem como a carência de dados locais, julgamos relevante um estudo de prevalência, visando conhecer a magnitude do problema em nosso meio. Este trabalho foi realizado com o objetivo de determinar a prevalência da obesidade e sobrepeso em adolescentes de uma escola da rede pública do Recife, situada numa comunidade de baixa renda, visando uma possível intervenção no processo.

\section{Métodos}

Realizou-se um estudo de corte transversal na escola Magalhães Bastos, uma escola da rede pública, 
situada numa comunidade de baixa renda da cidade do Recife, Pernambuco, Brasil. A população do estudo foi composta de todos os alunos na faixa etária de 10 a 19 anos, matriculados naquela escola no ano letivo de 2000, perfazendo um total de 230 adolescentes. A hipótese do estudo era que as prevalências de sobrepeso e obesidade nessa população seriam elevadas.

Em relação ao tamanho da amostra adequado, considerando uma prevalência de 6,5\%,14 um power de $80 \%$ e um nível de significância de $5 \%$, encontramos um $n$ de 156. Contudo, decidimos estudar o total da população, ao invés de uma amostra, para dar mais consistência à análise.

Inicialmente, realizou-se um trabalho de sensibilização com a direção e professores da escola, no qual foram apresentadas as etapas e procedimentos necessários para coleta de dados. Dessa forma, nos foi cedida uma listagem contendo os nomes e datas de nascimento dos alunos, através da qual selecionaram-se aqueles que estavam incluídos na faixa etária de 10 a 19 anos. Os pais dos alunos foram informados sobre a pesquisa através de uma circular e assinaram um formulário de consentimento informado, autorizando a participação do filho. Foram excluídos do estudo aqueles alunos que não estavam presentes na escola durante os dias da coleta, assim como aqueles que não tiveram a autorização dos pais ou recusaram-se a participar da pesquisa. O estudo foi aprovado pela Comissão de Ética em Pesquisa do Centro de Ciências da Saúde da Universidade Federal de Pernambuco. A coleta dos dados foi realizada no período de setembro a dezembro de 2000 , sendo os dados anotados em formulário próprio. Obtivemos uma perda de aproximadamente 8\% (19 alunos).

Os examinadores receberam um treinamento prévio para a realização das medidas antropométricas. Para aferição do peso, utilizou-se uma balança calibrada, da marca Filizola, com capacidade de $150 \mathrm{~kg}$, registrando-se o peso com uma precisão de $0,1 \mathrm{~kg}$, conforme recomendação da Organização Mundial da Saúde (OMS). A balança estava localizada sobre uma superfície rígida e plana, e foi zerada antes de cada pesagem. O indivíduo permanecia de pé, no centro da plataforma da balança, olhando para a frente ao ser pesado.

A estatura foi medida com o auxílio do antropômetro da mesma balança. Durante a medição, o indivíduo permanecia ereto, em inspiração profunda, com a cabeça posicionada de modo que o Plano de Frankfurt (o qual passa pela órbita e pelo meato auditivo externo) ficasse horizontal, joelhos esticados, pés juntos, braços soltos ao lado do corpo, com as palmas das mãos voltadas para as coxas. Se a leitura caísse entre dois valores, era anotado o menor.6,15 A estatura foi registrada com uma precisão de $0,1 \mathrm{~cm}$, como recomenda a WHO. 8 Peso e estatura foram medidos em duplicata, utilizando-se a média dos valores na análise dos dados.

Os dados foram processados e analisados com o auxílio do software Epi-Info (versão 6.0). Criou-se um banco de dados, contendo o nome do aluno, sua data de nascimento, peso, estatura e data em que foram efetuadas as medidas antropométricas. Realizou-se dupla entrada dos dados, bem como um processo de validação.

A partir desses dados primários, o IMC foi calculado e os indivíduos foram classificados segundo os percentis do IMC. Definiu-se sobrepeso como IMC igual ou superior ao percentil 85 e inferior ao percentil 95, para idade e sexo. 8 Obesidade foi definida como IMC igual ou superior ao percentil 95, para idade e sexo. ${ }^{8}$ Foi utilizada como população de referência a população americana estudada na National Health and Nutrition Examination Survey I (NHANES I), recomendada pela WHO. ${ }^{8}$

As prevalências de sobrepeso e obesidade foram calculadas pelo método de proporção, para cada um dos sexos, e comparadas através do teste de quiquadrado, com nível de significância de 5\% (p < $0,05)$. Foram também calculados os intervalos de confiança de $95 \%$ em torno de cada prevalência.

\section{Resultados}

Foram estudados 211 adolescentes, na faixa etária de 10 a 19 anos, dos quais $122(57,8 \%)$ eram sexo masculino e $89(42,2 \%)$ do sexo feminino. A Tabela 1 apresenta a distribuição dos estudantes, segundo o sexo e percentil do IMC. A prevalência de sobrepeso foi de $6,2 \%$ (IC95\% = 3,3 a 10,3\%), enquanto que a prevalência da obesidade foi de 5,7\% (IC95\% = 3,0 a 9,7).

Não houve diferença estatisticamente significante nas prevalências de sobrepeso e obesidade entre os sexos. A prevalência de sobrepeso no sexo masculino foi de $6,6 \%$ (IC95\% = 2,9 a 12,5\%) e, no feminino, $5,6 \%$ (IC95\% = 1,8 a 12,6\%) ( $\mathrm{p}=0,99)$. A prevalência de obesidade foi de $6,7 \%$ (IC95\% = 2,5 a $14,1 \%$ ) entre as adolescentes femininas e de $4,9 \%$ (IC95\% $=1,8$ a $10,4 \%)$, no sexo masculino ( $\mathrm{p}=$ $0,79)$. 
Distribuição de freqüência dos adolescentes, segundo sexo e percentil do Índice de Massa Corporal (IMC). Recife, Pernambuco, Brasil, 2000.

\begin{tabular}{|c|c|c|c|c|c|c|}
\hline \multirow{3}{*}{$\begin{array}{c}\text { IMC } \\
\text { (percentil) }\end{array}$} & \multicolumn{4}{|c|}{ Adolescentes } & & \\
\hline & \multicolumn{2}{|c|}{ Masculino } & \multicolumn{2}{|c|}{ Feminino } & \multicolumn{2}{|c|}{ Total } \\
\hline & $\mathrm{n}$ & $\%$ & $n$ & $\%$ & $\mathrm{n}$ & $\%$ \\
\hline$<85$ & 108 & 88,5 & 78 & 87,6 & 186 & 88,1 \\
\hline$\geq 85$ e $<95 *$ & 8 & 6,6 & 5 & 5,6 & 13 & 6,2 \\
\hline$\geq 95 * *$ & 6 & 4,9 & 6 & 6,7 & 12 & 5,7 \\
\hline Total & 122 & 100,0 & 89 & 100,0 & 211 & 100,0 \\
\hline
\end{tabular}

* Sobrepeso, ** Obesidade

\section{Discussão}

A escassez de estudos de prevalência para sobrepeso e obesidade na adolescência, associada à falta de unanimidade nos critérios de diagnósticos dessas condições, têm dificultado obter-se uma noção geral da real situação nutricional desse grupo etário.

Diversos métodos têm sido desenvolvidos para medir a gordura corporal, incluindo a densitometria, ultrassonografia, a medida da água e do potássio corpóreos, entre outros. Esses são, em geral, métodos caros, demorados e que não estão largamente disponíveis. As medidas antropométricas são uma alternativa simples, não invasiva, rápida e barata, que vêm sendo amplamente empregadas na clínica e em estudos epidemiológicos. 2,11

Vários autores têm demonstrado que o IMC consiste numa medida de adiposidade válida em crianças e adolescentes, utilizando como padrão ouro métodos mais sofisticados. Segundo Dietz,11 o IMC é o método mais adequado para avaliação do sobrepeso em crianças e adolescentes na rotina clínica e em saúde pública. De acordo com esse autor, o IMC consiste num índice adequado quanto aos critérios de precisão e acurácia. Dietz ${ }^{11}$ ressalta inclusive que a validade do IMC tem sido demonstrada não apenas do ponto de vista de "validade de medida", em estudos nos quais o IMC foi comparado com outros métodos mais acurados de medida da adiposidade, como também tem sido demonstrada sua "validade clínica", ou seja, de sua associação com diversas condições mórbidas.

Pietrobell et al. ${ }^{9}$ utilizaram a dual-energy x-ray absorptiometry (DEXA) como padrão ouro para avaliar se o IMC seria uma medida de adiposidade válida em crianças e adolescentes. Estudando indiví- duos de cinco a 19 anos, esses autores observaram que o IMC estava fortemente associado com a gordura corporal total e com o percentual de gordura corporal. Garrow e Webster ${ }^{10}$ observaram que o IMC fornece dados bastante acurados, tomando como padrão ouro uma média das estimativas de gordura corporal obtidas através da medida da densidade, da água e do potássio corpóreos.

Em nossa casuística, verificamos prevalências de $6,2 \%$ para sobrepeso e $5,7 \%$ para obesidade. Von der Heyde et al., 16 em 1996, encontraram prevalência de $11,2 \%$ para sobrepeso e $4,4 \%$ para obesidade em adolescentes com idade de 12 a 18 anos incompletos, moradores de 68 bairros de Curitiba. Castro et al., 14 em 1999, relataram prevalências de sobrepeso e obesidade de $10,4 \%$ e $6,4 \%$, respectivamente, entre adolescentes do Rio de Janeiro, sendo evidenciada uma tendência estatisticamente significante de crescimento da obesidade com o aumento da escolaridade materna. Salles et al.,17 em Florianópolis, avaliaram a prevalência de obesidade em estudantes na faixa etária de 10 a 17 anos, observando uma maior prevalência nas escolas da rede pública $(13,1 \%)$ do que na rede privada $(7,6 \%)$. Os autores citados utilizaram as mesmas definições de sobrepeso e obesidade por nós adotadas.

Em Recife, Balaban e Silva18 encontraram prevalências de sobrepeso e obesidade de $20,0 \%$ e $4,2 \%$, respectivamente, em adolescentes de uma escola da rede privada. Nesse estudo, foram considerados como portadores de sobrepeso os adolescentes com IMC igual ou superior ao percentil 85, para idade e sexo e, como obesos, aqueles com IMC e espessura da prega triciptal iguais ou superiores ao percentil 85.

As prevalências de sobrepeso e obesidade nos 
países desenvolvidos são maiores do que as observadas em nosso estudo. Com relação aos adolescentes americanos, Troiano et al. 19 relataram uma prevalência de sobrepeso de $21,7 \%$, sendo semelhante à prevalência entre os adolescentes masculinos $(22,0 \%)$ e femininos $(21,4 \%)$. A prevalência de obesidade relatada por Gortmaker et al.20 foi de $21,9 \%$, sendo maior no sexo feminino $(25,5 \%)$ do que no masculino (18,3\%).

Nos Estados Unidos, de 1976 a 1987, a prevalência da obesidade e da superobesidade em jovens de 12 a 21 anos aumentou em 64\%.3 A diminuição na prática de atividades físicas desempenha um importante papel na tendência crescente da prevalência da obesidade. Outro problema que vem a favorecer essa tendência é a marcante mudança de hábitos alimentares que tem acompanhado o processo de modernização industrial. ${ }^{3,21}$

Monteiro et al.,21 com base em dois grandes estudos transversais de abrangência nacional (de 1974 e 1989), documentaram o processo de transição nutricional no Brasil, constatando uma rápida substituição do problema da escassez pelo problema do excesso dietético. Nesse período de 15 anos, houve uma redução de cerca de $60 \%$ na prevalência de desnutrição e aumentos variáveis na de obesidade, nos diversos grupos etários e socioeconômicos.

\section{Referências}

1. Dietz WH. Periods of risk in childhood for development of adult obesity. What do we need to learn? J Nutr 1997; 127: 1884 S-6S

2. Zlochevsky ERM. Obesidade na infância e adolescência. Rev Paul Pediatr 1996; 14: 124-33.

3. Schonfeld-Warden N, Warden CH. Obesidade pediátrica: uma visão global da etiologia e do tratamento. Clin Pediatr Am Norte 1997; 2: .343-66.

4. Escrivão MAMS, Oliveira FL, Taddei JAAC, Lopez A. Obesidade exógena na infância e na adolescência. J Pediatr (Rio de Janeiro) 2000; 76 (Supl. 3): 305-10.

5. Must A. Morbidity and mortality associated with elevated body weight in children and adolescents. Am J Clin Nutr 1996; 63: 445-7.

6. Veiga GV, Sampei MA, Sawaya AL, Sigulem DM. Adaptação do critério antropométrico para avaliação do estado nutricional de adolescentes em dois níveis socioeconômicos no município de São Paulo. J Pediatr (Rio de Janeiro) 1992; 68: 26-33.

7. Siquieri R. Epidemiologia da obesidade. Rio de Janeiro: Ed. Universidade do Rio de Janeiro; 1998.

Anjos 22 analisou as medianas do IMC de brasileiros de 10 a 20 anos de idade, no Nordeste e Sudeste do país, entre 1974 e 1997, encontrando uma tendência secular positiva.

Os achados do presente estudo, somados às evidências de outros estudos citados, indicam que a crença de que a obesidade seja um problema relevante apenas nos países desenvolvidos e em populações de alta renda parece equivocada. A prevalência da obesidade vem aumentando de forma significativa também nos países em desenvolvimento que experimentam rápidas e intensas transformações em seu padrão de crescimento econômico e estrutura demográfica.

Devido à sua crescente prevalência, sua persistência ao longo da vida e sua associação com diversas condições mórbidas, a obesidade posiciona-se atualmente como um dos grandes problemas nacionais de saúde. A educação, o nível de informação da população sobre os riscos associados ao ganho de peso excessivo, bem como fatores culturais e comportamentais, tendem a assumir papel de destaque na epidemiologia da obesidade. Portanto, mostra-se necessária a implantação de programas de educação alimentar e incentivo à prática de atividades físicas nas escolas, assim como uma revisão das prioridades e estratégias de intervenção da saúde pública brasileira.

8. WHO (World Health Organization). Physical status: the use and interpretation of anthropometry: report of a WHO Expert Committee. Geneva: WHO: 1995.

9. Pietrobelli A, Faith MS, Allison DB, Gallagher D, Chiumello G, Heymsfield SB. Body Mass Index as a measure of adiposity among children and adolescents: a validation study. J Pediatr 1997; 132: 204-10

10. Garrow JS, Webster J. Quetelet's index (W/H 2) as a measure of fatness. Int J Obes 1985; 9: 147-53.

11. Dietz WH. Use of the Body Mass Index (BMI) as a measure of overweight in children and adolescents. J Pediatr 1998; 132: 191-3.

12. Must A, Dallal GE, Dietz WH. Reference data for obesity 85th and 95th percentiles of Body Mass Index (wh/ht 2) and triceps skinfold tickness. Am J Clin Nutr 1991; 53 : 839-46.

13. Himes JH, Dietz WH. Guidelines for overweight in adolescent preventive services: recommendations from na Expert Committee. Am J Clin Nutr 1994; 59: 307-16.

14. Castro IRR, Engstrom EM, Anjos LA, Azevedo AM, Silva CS. Perfil nutricional dos alunos da rede municipal de 
educação da cidade do Rio de Janeiro. In: Oliveira JED, Lamounier JA, Assis AMO, Berezovsky MW, Portella Jr AO. Obesidade e anemia carencial na adolescência: simpósio. São Paulo: Instituto Danone; 2000. p 231-2.

15. Gibson RS. Principles of nutritional assessment. Oxford: Oxford University Press; 1990.

16. Von der Heyde MED, Amorim STSP, Lang RMF, Von der Heyde R. Perfil nutricional de adolescentes da cidade de Curitiba. In: Oliveira JED, Lamounier JA, Assis AMO, Berezovsky MW, Portella Jr AO. Obesidade e anemia carencial na adolescência: simpósio. São Paulo: Instituto Danone; 2000

17. Salles RK, Kazapi IA, Di Pietro PF. Ocorrência de obesidade em adolescentes da rede de ensino do município de Florianópolis. In: Oliveira JED, Lamounier JA, Assis AMO, Berezovsky MW, Portella Jr AO. Obesidade e anemia carencial na adolescência: simpósio. São Paulo: Instituto Danone; 2000

18. Balaban G, Silva GAP. Prevalência de sobrepeso e obesidade em crianças e adolescentes de uma escola da rede privada de Recife. J Pediatr (Rio de Janeiro) 2001; 77 : 96-100.
19. Troiano RP, Flegal KM, Kukzmarski RJ, Campbell SM Johnson CL. Overweight prevalence and trends for children and adolescents - the National Health and Nutrition Examination Surveys, 1963 to 1991. Arch Pediatr Adolesc Med 1995; 149: 1085-91.

20. Gortmaker SL, Dietz WH, Sobol AM, Wehler CA. Increasing pediatric obesity in the United States. Am J Dis Child 1987; 141: 535-40.

21. Monteiro CA, Mondini L, Souza ALM, Popkin BM. Da desnutrição para a obesidade: a transição nutricional no Brasil. In: Monteiro CA. Velhos e novos males da saúde no Brasil - A evolução do país e de suas doenças. São Paulo: Hucitec; 1995. p. 247-55.

22. Anjos LA. Tendência secular do índice de massa corporal de adolescentes brasileiros do Nordeste e Sudeste entre 1974 e 1997. In: Oliveira JED, Lamounier JA, Assis AMO, Berezovsky MW, Portella Jr AO. Obesidade e anemia carencial na adolescência: simpósio. São Paulo: Instituto Danone; 2000. p. 89-95. 